\title{
Postural adjustments associated with rapid voluntary arm movements 1. Electromyographic data
}

\author{
WG FRIEDLI, * M HALLETT, SR SIMON \\ From the Gait Analysis Laboratory of the Rehabilitation-Engineering Center of Harvard-MIT, Section of \\ Neurology, Department of Medicine, Brigham and Women's Hospital, Department of Orthopedic Surgery, \\ Children's Hospital Medical Center and Departments of Neurology and Orthopedic Surgery, Harvard \\ Medical School, Boston MA, USA
}

SUMMARY Normal subjects made bilaterally symmetric rapid elbow flexions or extensions ("focal movement") while free standing or when supported by being strapped to a firm wall behind them (different "postural set"). In some trials a load opposed the movement two thirds of the way into its course. Electromyographic activity in leg and trunk muscles ("associated postural adjustments") demonstrated specific patterns for each type of movement. Activity in these muscles began prior to activity in the arm muscles and demonstrated a distal-to-proximal order of activation. The EMG patterns were characterised by alternating activity in the antagonist pairs similar to the triphasic pattern seen in the arm muscles. When the movement type was changed change of the pattern of the postural muscles occurred over several trials. It is concluded that the associated postural adjustments are pre-programmed motor activity linked to the focal movement, specific for the focal movement including anticipated events and the postural set.

Regulation of man's upright posture involves the complex interaction of different systems, operating in both feedback and feedforward modes of control. Feedback mechanisms have been siudied by analysing postural responses to direct perturbations of the body relationship to the ground ${ }^{1-7}$ and also perturbations of a limb where movements of the body would be expected to occur but had not yet occurred. ${ }^{8}$ Less attention has been paid to the preparatory processes for voluntary motor activity. The preparation of a sequence of activity in different muscles, which we will call a postural pattern, associated with a limb displacement requires taking into account the physical characteristics of the limb movement and the postural requirements. EMG activity in postural muscles which is characteristic for a particular situation has been seen in both primates $^{9-12}$ and

\footnotetext{
${ }^{*}$ Current address and address for reprint requests: Walter G. Friedli, Neurologisce Universitätsklinik, Kantonsspital 4031 Basel, Switzerland.
}

Received 1 September 1983 and in revised form 28 November 1983

Accepted 22 December 1983

A preliminary account of some of the findings were presented at Xth International Congress of Electroencephalography and Clinical Neurophysiology, Kyoto, September 1981 humans; ${ }^{13-17}$ However, the physiological principles which determine these patterns have not been fully clarified.

The objective of the present experiments was the further examination of the central linkage between rapid voluntary arm movements and the postural adjustments associated with them. For several reasons, we have chosen a fast elbow movement, performed in upright standing subjects. First, a rapid arm movement causes more postural disturbance than a slow one. Second, since there has already been evidence that the EMG pattern in the agonist and antagonist of a fast arm movement is preprogrammed, ${ }^{18-20}$ the EMG activity in the postural muscles may also be pre-programmed. Third, the EMG pattern of a fast arm movement is distinctive with a triphasic pattern of sequential bursts of activity in the agonist, then in the antagonist and then in the agonist again. ${ }^{19-25} \mathrm{~A}$ similar distinctive pattern might also characterise postural activity.

\section{Methods}

The basic experiments were performed on 17 healthy male and female volunteers ranging in age from 25 to 65 years. In each session, the subject stood upright on a force platform (fig 1). The subject was instructed to stand relaxed, place equal weight on each foot, and look at a light 


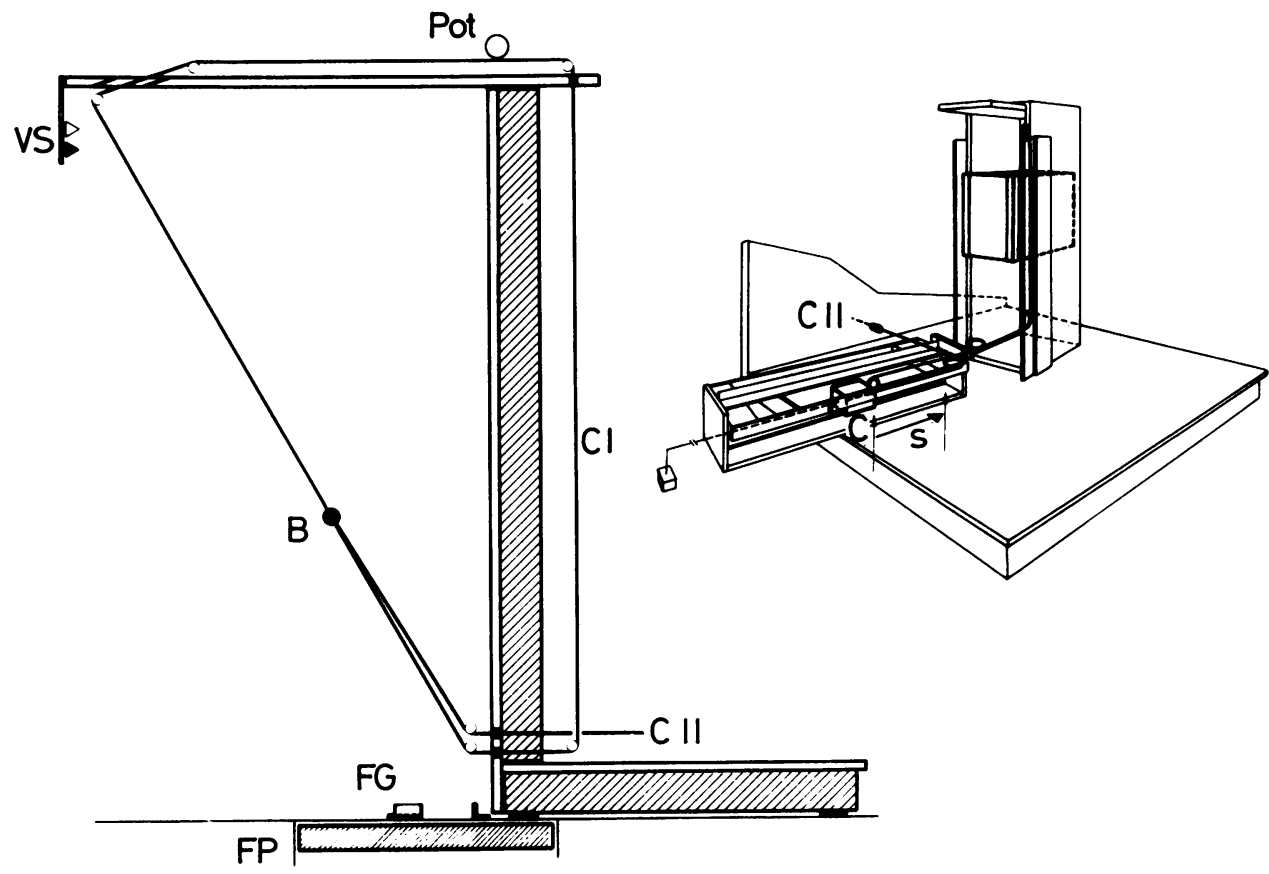

Fig 1 Experimental apparatus: the subject stands on the force plate (FP). Feet are placed on the plate in accordance to a foot guide $(F G)$ which insures symmetrical foot position. Commanded by the visual signal $(V S)$, the subject moves the metal bar $(B)$ which drives the potentiometer (Pot) by pulling on the attached nylon cord (CI). On some trials the body was supported by strapping the subject to the firm wall. Load is applied in some trials by connecting the mass $(M)$ to $B$ by a second nylon cord (C II) which is guided by means of pullies over a moving cart $(C) . M$ is pulled up after $C$ has covered the span $S$, which is adjusted for each subject so that the load comes into play after $60^{\circ}$ of movement.

mounted in front of the platform. With the forearm supinated the subject grasped a horizontal metal bar. In every experimental condition, with the upper arm vertical and held adjacent to the trunk, the subject was asked to perform a bilaterally symmetric fast elbow movement, consisting of moving the bar as rapidly as possible up to $90^{\circ}$ of elbow flexion, or down from $90^{\circ}$ of flexion to full extension. The subject was instructed to pay more attention to the velocity of the movement than to accuracy. Subjects were given several trials of practice at the beginning of the session in order to familiarise themselves with the system.

Four different experimental conditions were investigated with each subject:

(1) Unsupported-unloaded: The free-standing subject performed rapid elbow flexions without additional load.

(2) Unsupported-loaded: A load of $1 \mathrm{~kg}$ was added to the cable attached to the bar at $60^{\circ}$ of elbow movement.

(3) Supported- unloaded: The subject was strapped to a firm wall with belts and performed the movement without load perturbation.

(4) Supported-loaded.

Elbow extensions without any load perturbation were investigated with 10 of the subjects in an unsupported state; two of them were also investigated in the supported state.
Each session was started with one of the unloaded conditions: the sequence of conditions thereafter was arbitrary. At least 10 , maximally 15 movements, were made by the subjects for each condition. The interval between two subsequent movements within each condition was at least 30 seconds. The subject relaxed until the experimenter informed him that a new trial would begin. The signal to make the movement was turning on of the light in front of the subject.

With two subjects an experiment was designed in order to investigate the effect of the extent of arm movement on postural responses. The subjects performed rapid elbow flexions up to $50^{\circ}$ and $70^{\circ}$ in addition to the usual $90^{\circ}$ in the unsupported-unloaded condition.

\section{Recording system}

Using the same equipment as for the investigation of gait in the Gait Analysis Laboratory' ${ }^{27}$ four types of data were gathered in these experiments: the trajectory of the arm movement, electromyograms from arm as well as from various postural muscles, the foot-floor reaction forces, and high speed cinematography of the subject. The present paper is confined to electromyographic data while the examination of foot-floor reaction forces and motion data will be the subject of a subsequent paper. 
The arm movement in terms of position of the bar was measured with a precision multi-turn potentiometer, driven by a nylon cord which was fastened to the bar.

Electromyographic activity was recorded using surface electrodes placed on the right side of the body over the motor points of biceps brachii, triceps brachii, quadriceps, biceps femoris (long head), tibialis anterior, medial gastrocnemius, thoracolumbar erector spinae and rectus abdominis above the navel. In one subject additional electrodes were placed over deltoidei anterior and posterior. The electrodes were connected to specially designed small preamplifiers (Input impedance $>1 \mathrm{M}$ ohm; gain 450; common mode rejection ratio $105 \mathrm{~dB}$; band width $0-10$ $\mathrm{kHz}$ ) which in turn were connected to variable gain amplifiers with band pass of 15 to $100 \mathrm{~Hz}$.

At the beginning of each session the subject was asked to make a maximal tonic contraction for each of the tested muscles. EMG activity relating to a movement could be compared to this maximal effort in order to obtain information about the approximate percent effort of the muscle in the movement.

Potentiometer, electromyographic and force-plate signals were continuously transmitted to a PDP 11-34 computer and sampled every 2 milliseconds for a total of 1 second. The photic command signal to move was given at the time of initiation of data collection.

\section{Processing of electromyographic data}

For best visualisation, computer programs altered the gain of the EMG signals to allow them to be plotted as large as possible within a fixed coordinate size. The amplification is represented by a "scale factor" ranging from 1 to 10 for each muscle tested. From scale factors alone comparisons of signal magnitude for one muscle of the same subject in different trials was possible.

Electromyographic activity was full-wave rectified and plotted on a scale of 0.5 inch $(12.5 \mathrm{~mm})$ per $10 \mathrm{~ms}$. From these plots, a signal amplitude of one fifth of full scale was required to define the onset of burst activity. The end of the burst was defined when the last activity of the burst had decreased in amplitude to less than one fifth of full scale, while still being "connected" to the burst by a period of less than $20 \mathrm{~ms}$. The magnitude of EMG activity under different conditions in the same subject was compared by averaging the signals of all the trials within one condition. The onset of movement given by the potentiometer signal was used as the reference point. Subtracting the averaged data from one condition from that of another condition produced differences in magnitude of activity between the conditions in the same subject. Electromyographic activity was also filtered by means of a symmetrical boxcaraveraging of $10 \mathrm{~ms}$ window-width for smoother graphical display of the signals.

\section{Results}

\section{THE BURST PATTERNS}

EMG activity in the biceps and triceps was characterised by a pattern of alternating bursts similar to the "triphasic" pattern underlying rapid arm movements of less extent in the horizontal plane. In flexion movements a first burst of activity in biceps brachii (agonist) was followed by a period of relative silence during which there was a burst in triceps brachii (antagonist), followed by a second agonist burst. Under the experimental condition of upright stance burst patterns were found not only in the arm but also in the different trunk and leg muscles (fig 2). We will define the agonist of a pair of antagonist postural muscles according to which of the two muscles was activated earlier.

\section{Unsupported-unloaded condition}

In rapid elbow flexions without body support erector spinae and biceps femoris are agonists, while rectus abdominis and quadriceps femoris are their antagonists respectively (fig $2 \mathrm{~A}$ ). Gastrocnemius and tibialis anterior seemed to play a minor role as postural muscles during rapid elbow movements since their phasic activity was either weak or not recorded at all. When the "triphasic" pattern was seen in these

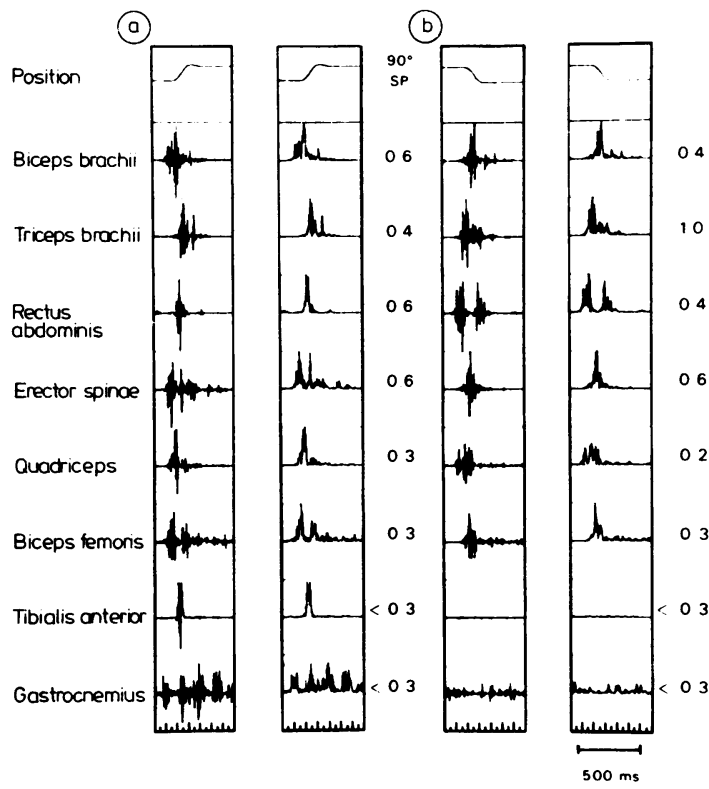

Fig 2 Examples of EMG activity showing multiple-burst patterns in arm and postural muscles with fast elbow movements during upright stance. Raw and rectified EMG activity for two single trials are shown. A flexion movement $(A)$ is compared to extension movement $(B)$. Notice muscles of antagonist pairs changing their roles in the different movements. In contrast to those in subsequent figures scale factors are relative to maximal force, that is the numbers represent the ratio of scale factors for the phasic activity during the ballistic movement and for the tonic activity with maximal contraction (see text). The potentiometer signal at the top represents the movement of the bar. Data recording starts with the onset of the visual command to move. 


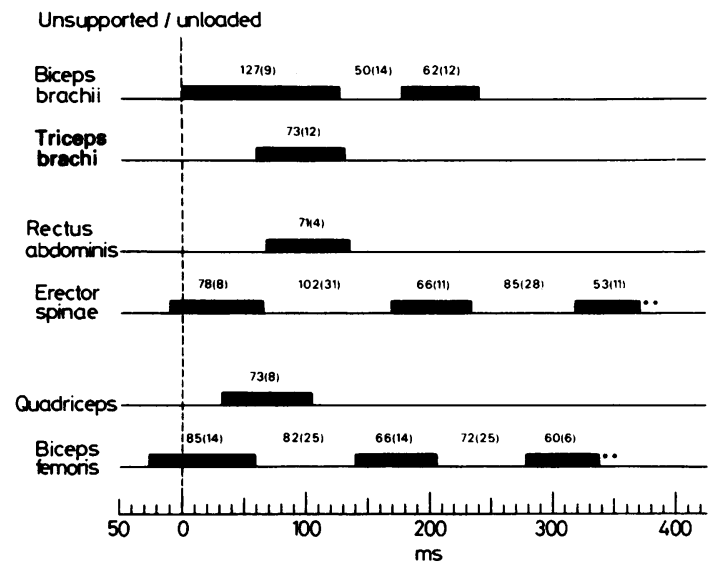

Fig 3 Averaged EMG pattern of three antagonist muscle pairs with elbow flexion without load perturbation in the unsupported state. The beginning of EMG bursts is related to the onset of biceps brachii as the prime mover (Time 0). Numbers represent mean durations (standard deviations) of bursts or intervals respectively from 10 subjects. ${ }^{* *}$ indicates bursts which are present in fewer subjects.

muscles, gastrocnemius was the agonist and tibialis anterior the antagonist.

Time relationships between the different muscles were provided by measurements of burst onset times in single trials. The beginning of the bursts in arm and postural muscles was related to the onset time of biceps brachii as the prime mover. The latter preceded the onset of arm movement itself by 75 to 85 $\mathrm{ms}$, measured by the potentiometer signal (by comparing the onset of movement in film data with the potentiometer signal, the latter was found to be delayed by 20 to $45 \mathrm{~ms}$ ). For all the subjects the time order of agonists was consistent, biceps femoris being the first, erector spinae the second muscle, followed by biceps brachii as the prime mover (fig 3 ). The antagonists followed the same general order, although there was only a small difference between the onset times of rectus abdominis and triceps brachii. The subject's instruction was to make a movement as rapidly as possible and not specifically to make the reaction time as short as possible, although in general subjects made their movements promptly after the visual command. Regardless of markedly variable reaction time, there was only little variation of relative timing (table).

The durations of the first agonist bursts of the different muscles were in the same range ( $70-90 \mathrm{~ms})$ except for biceps brachii whose burst duration was well over $100 \mathrm{~ms}$. There were normally two bursts in biceps brachii and two or three bursts in the postural agonist muscles. The durations of later bursts were briefer than the initial bursts and the intervals between subsequent bursts in the same muscle became progressively shorter. The antagonist bursts had similar durations (60-80 ms) and were occasionally followed by smaller later bursts in the silent period of the corresponding agonist muscle. Whereas in postural muscles the first agonist burst usually terminated at about the time of onset of the antagonist burst, there was clear overlapping in time of antagonist activity with the first agonist burst in the arm muscles.

Magnitude of EMG activity in the unsupported unloaded condition was estimated by comparing activity produced by maximal tonic contraction of each muscle at the beginning of the session. This comparison was more reliable for limb than for trunk muscles since it was difficult for subjects to maximally activate trunk muscles voluntarily. Similar results were obtained for all subjects and the values for one subject can be noted in fig 2 . Activity in the arm muscles was strong in order to make the rapid movement. Activity was similarly powerful for the trunk muscles, less for proximal and only minimal for distal leg muscles. For other conditions, magnitude of activity will be compared relative to this basic condition.

\section{Unsupported-loaded condition}

In this condition the rapid elbow flexion was disturbed by a load at $60^{\circ}$ of movement. The postural muscles kept their roles as agonists and antagonists (fig 4).

The initial bursts of the postural agonists were earlier with respect to biceps brachii than in the unloaded condition (fig 5). This effect was more pronounced for biceps femoris than for erector spinae. The burst durations of the postural muscles did not change whereas the first agonist burst of biceps brachii was significantly longer in the condi-

Table Latencies (LAT) between the onset of postural and prime mover bursts are compared to their reaction time (RT) with respect to the visual command for one subject.

\begin{tabular}{|c|c|c|c|c|c|}
\hline \multirow[t]{2}{*}{ Condition } & \multicolumn{2}{|c|}{ Biceps femoris $(B F)$} & \multicolumn{2}{|c|}{ Erector spinae $(E S)$} & \multirow{2}{*}{$\frac{\text { Biceps brachii }(B B)}{R T_{B B}}$} \\
\hline & $R T_{B F}$ & $L A T_{B F}$ & $R T_{E S}$ & $L A T_{E S}$ & \\
\hline $\begin{array}{l}\text { Unsupported-unloaded } \\
\text { Unsupported-loaded }\end{array}$ & $\begin{array}{l}199 \pm 101 \\
289 \pm 146\end{array}$ & $\begin{array}{l}32 \pm 8 \\
51 \pm 10\end{array}$ & $\begin{array}{l}217 \pm 107 \\
319 \pm 148\end{array}$ & $\begin{array}{l}17 \pm 4 \\
26 \pm 7\end{array}$ & $\begin{array}{l}237 \pm 113 \\
349 \pm 146\end{array}$ \\
\hline
\end{tabular}




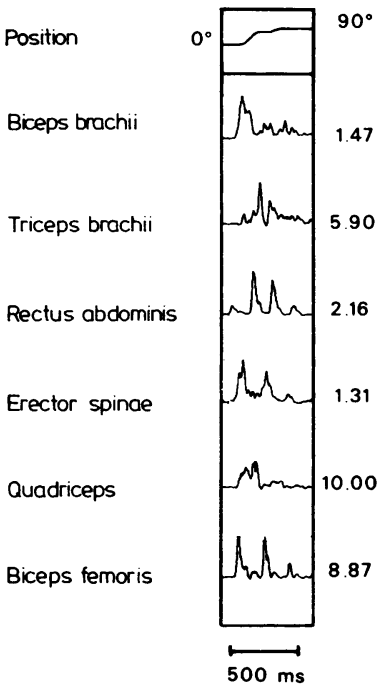

Fig 4 Example of EMG activity with elbow flexion with the un-supported -loaded condition. Single movement of one subject, with emg rectified and Boxcar-fittered. Notice the occurence of distinct later bursts in agonists and antagonists producing impressive alternating patterns. Movement of the bar is represented by the potentiometer signal. tion with load perturbation. For both erector spinae and biceps femoris the interval between the first and second burst was significantly longer compared to the condition without load perturbation ( $p<$ 0.001 ). Their second agonist bursts were aligned with each other as well as with the second burst of biceps brachii. In general, there were more later bursts in both arm and postural muscles, following each other with successively shorter intervals in between. Activity of postural antagonists was diminished. Corresponding to the longer interval between the first and second agonist burst the initial antagonist burst was delayed, shifted between the two agonist bursts, and often composed of two distinctive parts. Multiple antagonist bursts produced impressive alternating burst patterns in some of the subjects.

The magnitude of EMG activity was assessed by subtracting the averaged signals of the "unsupported-unloaded" from the "unsupportedloaded" condition (fig 6D). For biceps brachii as well as for the postural agonists positive differences resulted from this procedure, indicating more signal magnitude in the "loaded" condition.

\section{Supported-unloaded condition}

In this condition the subjects performed rapid elbow flexions while being strapped to the wall. Postural activity was reduced (fig 6B) and even second agon-

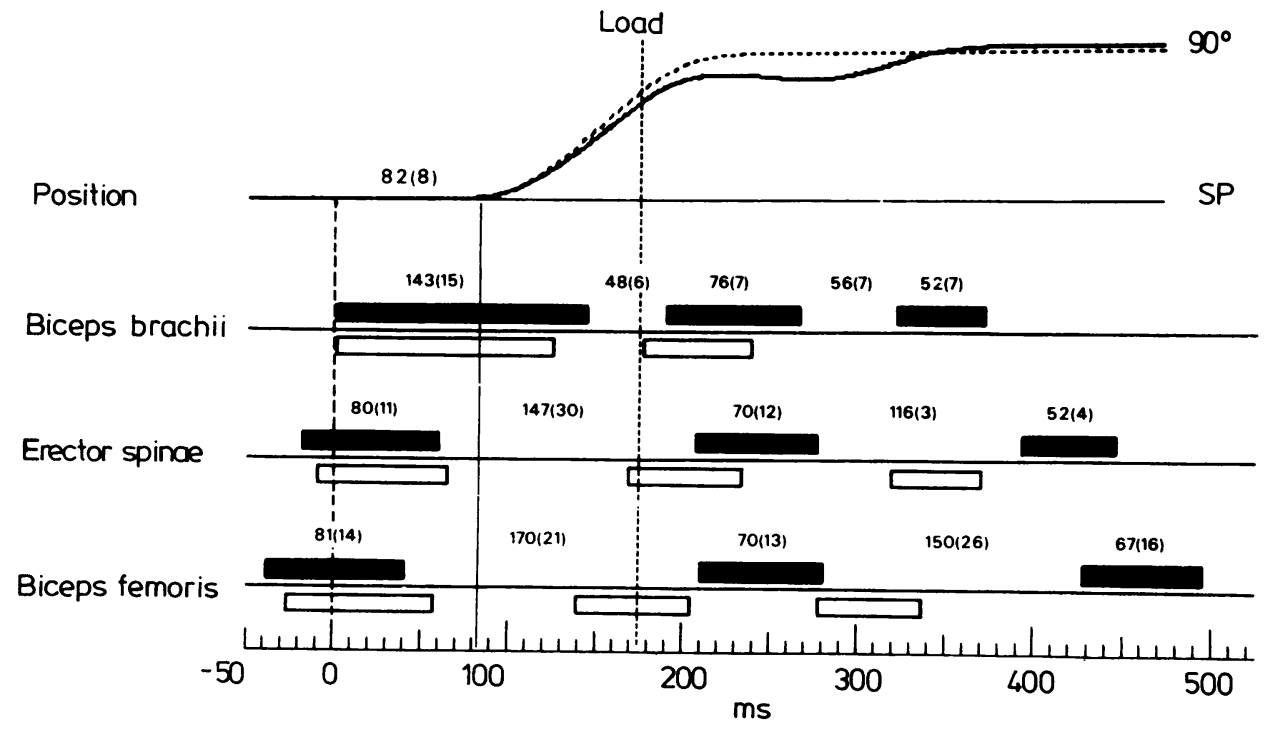

Fig 5 Average timing of EMG activity for the unsupported-loaded task (black bars) compared to the unsupported-unloaded task (white bars). The behaviour of the EMG patterns in the loaded circumstance differs from the unloaded situation not only after the load is added but also before it (see text). Averaged position records for the loaded and unloaded condition are superimposed (Data from 5 subjects). 


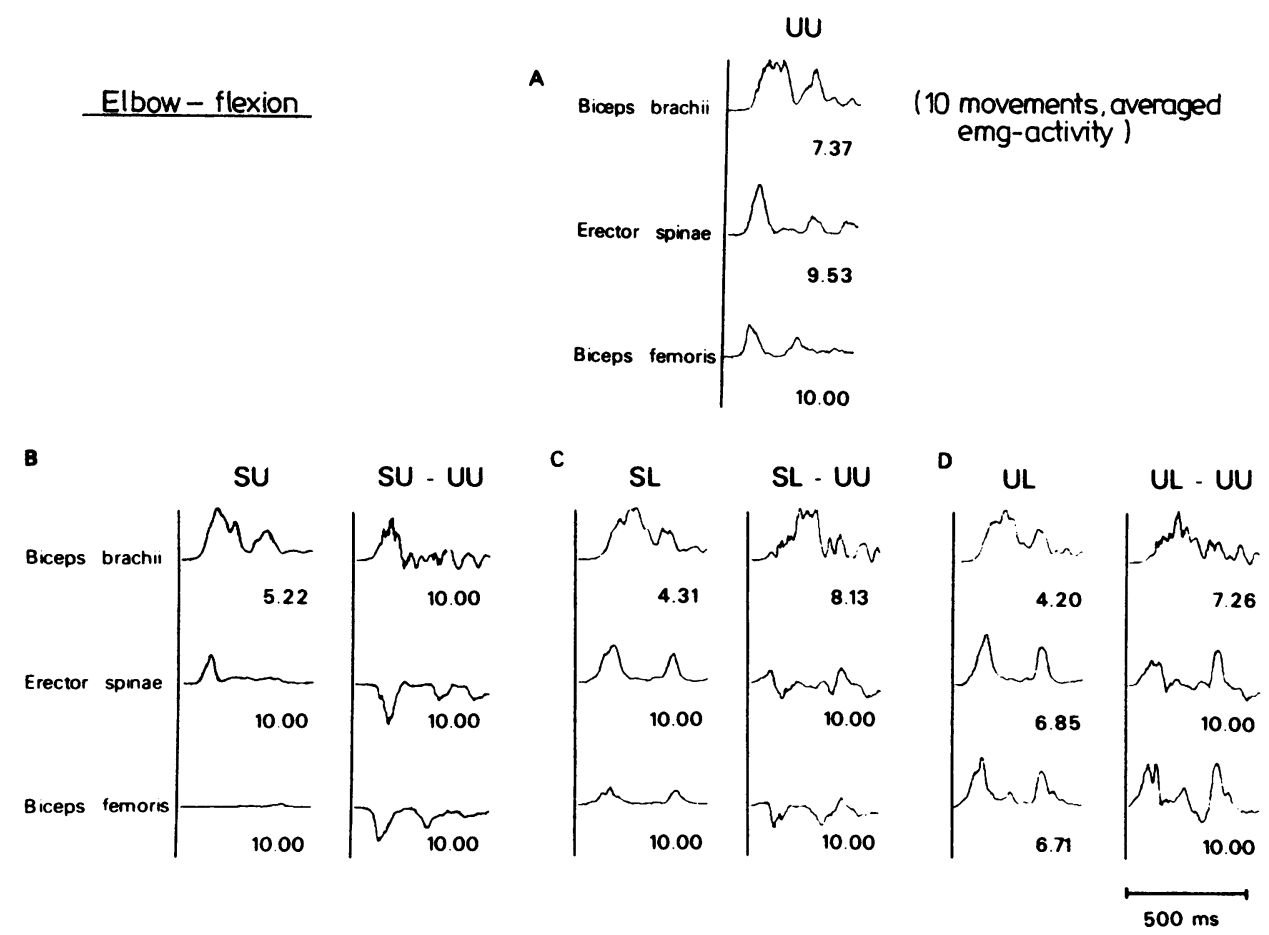

Fig 6 Average rectified-and-smoothed EMG activity from biceps brachii and postural agonists in the different conditions for one subject ( $U U=$ unsupported-unloaded; $S U=$ supported-unloaded; $S L=$ supported-loaded; $U L=$ unsupported-loaded). Subtraction of the basic condition $U U(A)$ from each of the others brings out the differences in magnitude clearly. A negative difference means that UU is larger (for example, erector spinae in B); a positive difference indicates that the other condition is larger (for example, erector spinae in D). Compare also the scale factors which are inversely related to the amplitude of averaged signals.

ist bursts might be absent. Additionally, the onset of postural agonists erector spinae and biceps femoris with respect to the beginning of prime mover activity was later compared to the situation without body support (fig 7A). The durations of the first agonist bursts as well as of the antagonists bursts in arm and postural muscles were comparable to those in the unsupported state without load perturbation.

\section{Supported-loaded condition}

The presence of load influenced activity in the supported condition similar to its influence in the unsupported state. There was again a longer duration of the first burst in biceps brachii in the loaded condition, whereas the postural muscles showed similar burst durations under both conditions. The onset of erector spinae was earlier with load similar to the situation without body support (fig 7B). The onset time of biceps femoris, however, was not consistent. The interval between the first and second burst in erector spinae was significantly lengthened $(p<0.001)$. There was also a longer interval in biceps femoris when the second burst could be recognised.

There was less postural activity in the supported-loaded than in the unsupportedunloaded condition, especially in the more distal muscles (fig 6C).

\section{ADDITIONAL EXPERIMENTAL CONDITIONS \\ 1. Elbow extensions}

Triceps brachii was the prime mover agonist and rectus abdominis and quadriceps were the postural agonists (fig 2B). The activities of both gastrocnemius and tibialis anterior were weak with extension movements and were even less frequently recorded than with elbow flexions. If present, tibialis anterior showed the agonist pattern whereas gastrocnemius was the antagonist. The onset of rectus abdominis as the postural agonist clearly preceded 
a Unloaded
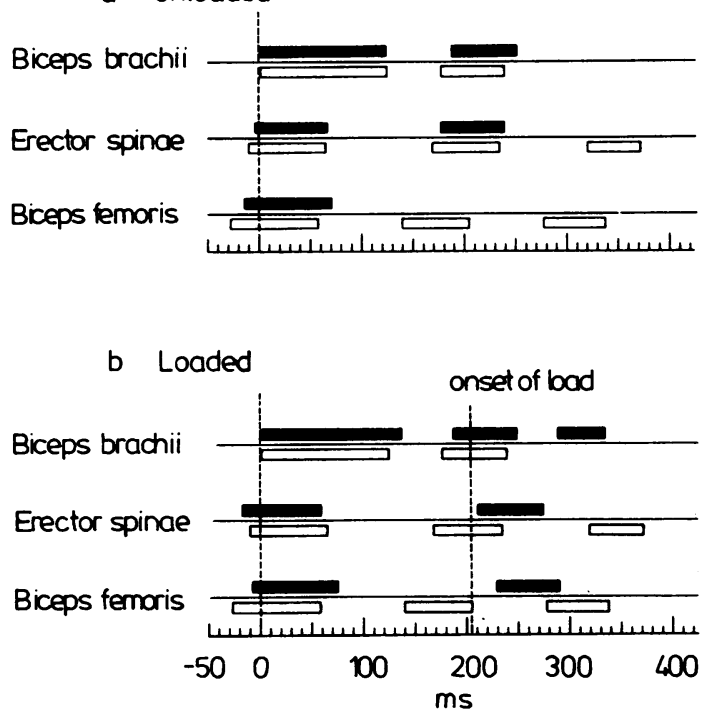

Fig 7 Average timing of EMG activity for the supported conditions (black bars) without (a) and with load perturbation (b) compared to the unsupported-unloaded task (white bars). Averaged data from 5 subjects. Note the increase of the interval between the first and second burst in postural muscles with load perturbation.

the beginning of the prime mover in all the subjects (fig 8). The onset times of quadriceps and biceps femoris were less consistent.

By comparing the burst durations of the principle muscles for extension and flexion movements the main difference was found in the arm muscles. Triceps brachii as the prime mover agonist had a

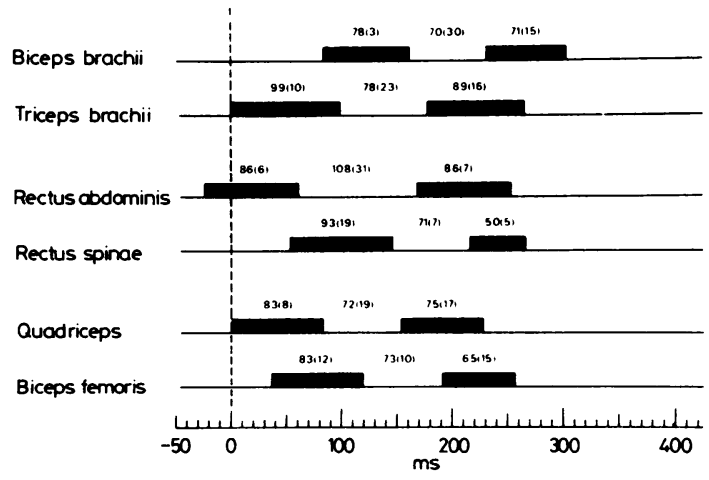

Fig 8 Average timing of EMG activity of 3 antagonist muscle pairs with elbow extension without body support from 5 subjects. Burst onset is related to the onset of triceps brachii as the prime mover. (Time 0). Note the burst pattern is not distinct in the distal antagonist pair. longer duration in extensions $(85-110 \mathrm{~ms})$ than it had as the antagonist. The burst of biceps brachii as the antagonist was significantly shorter than in flexion movements (60-80 ms). There was also a clearer triphasic pattern of the two arm muscles, the overlapping of antagonist activity with the first agonist burst being less pronounced in elbow extensions than in flexions. In all the subjects rectus abdominis and erector spinae showed a distinct alternating pattern while this phenomenon was frequently not seen in the thigh muscles.

Inhibition of antagonist activity preceding the onset of the first agonist burst in arm muscles as well as in the postural muscles was seen in some subjects (fig 9). The time order of the appearance of this inhibitory period in the different antagonists followed the same order of agonist activation which is from distal to proximal.

\section{Extent of arm movement}

Two subjects performed sets of elbow flexions with end points of $50^{\circ}$ and $70^{\circ}$ as well as $90^{\circ}$ (fig 10). In both the onset time of the first burst in postural agonists tended to be earlier with smaller movements. This behaviour was more evident for biceps brachii than for erector spinae. There was some delay of the second burst in the postural agonists in

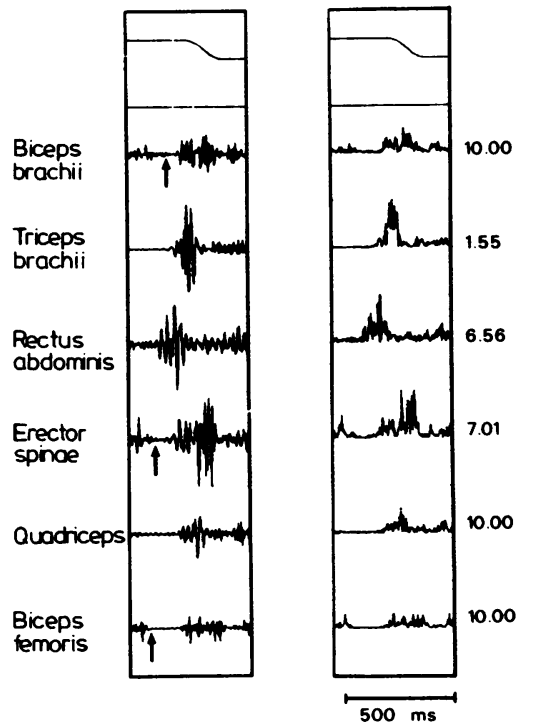

Fig 9 EMG activity for an elbow extension movement with an example of inhibition of antagonist background activity prior to the onset of the first agonist burst (arrows).

Antagonist inhibition is seen in arm and postural muscles, following the distal-to-proximal order. Raw data (left) and rectified emg activity (right) of a single trail. 


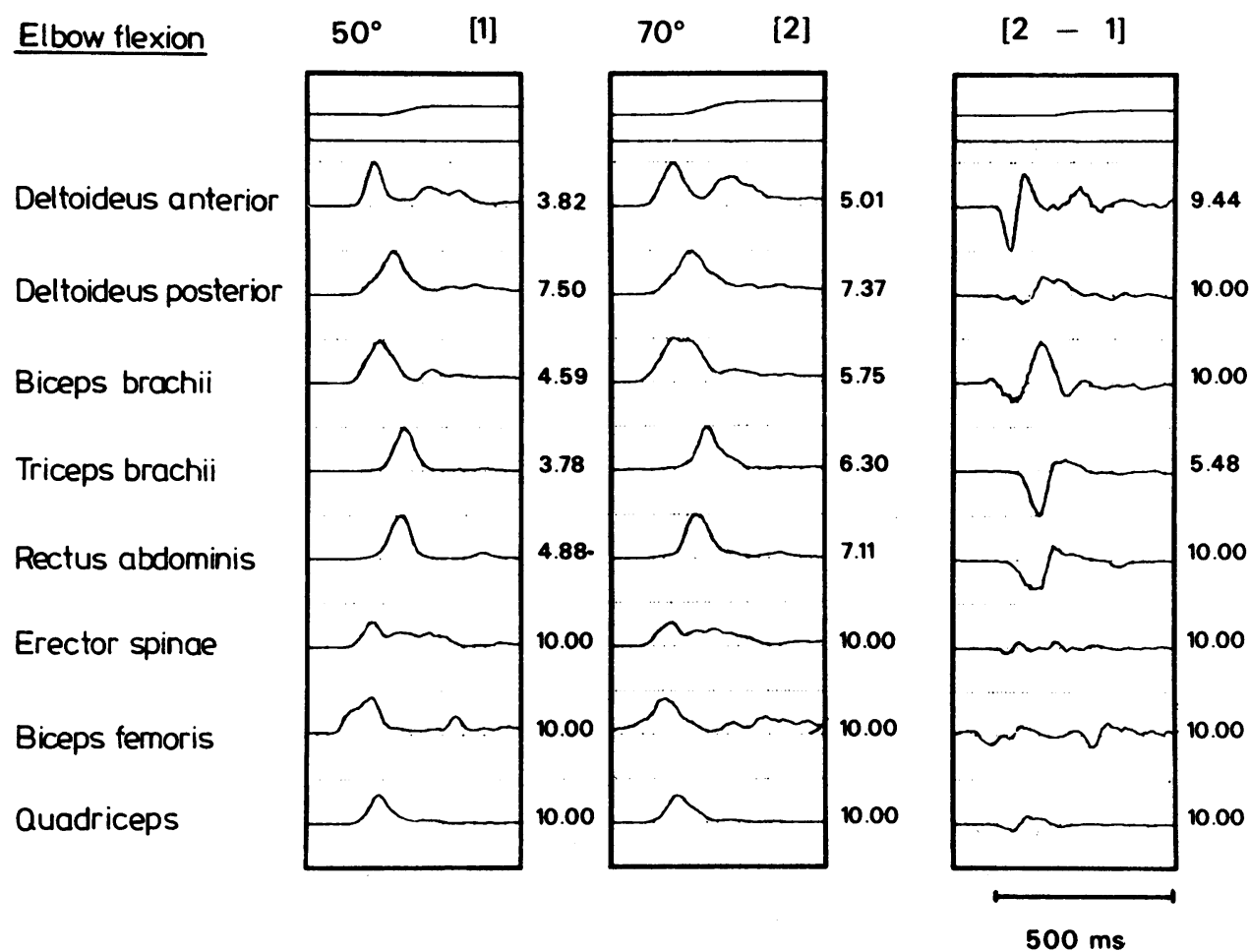

Fig 10 EMG activity associated with differing extent of elbow flexion. Records show average rectified-and-smoothed emg activity of 10 movements of $50^{\circ}(1)$ and $70^{\circ}(2)$ from one subject. Note the clear triphasic pattern of the two deltoid portions also recorded here (fixing the upper arm to the trunk). Since there are increased scale factors of triceps brachii and rectus abdominis with the larger movement, the subtraction of 1 from 2 (right column) reveals more magnitude of these muscles in flexion movements of lesser extent.

smaller movements. In the two subjects however, the data in respect of the onset of burst activity did not differ significantly to be conclusive enough. The first burst of biceps brachii was found to be lengthened proportional to the extent of elbow flexion from about 100 to $135 \mathrm{~ms}$, while otherwise the burst durations of arm and postural muscles were the same under different conditions. Triceps brachii and rectus abdominis both showed larger EMG magnitude in smaller movements. Although not significant due to the small magnitude of the averaged signals, the difference for quadriceps tended to be in the same direction.

\section{ADAPTATION}

The EMG activity in postural muscles, which has so far been analysed in averages of 10 or more trials, varies significantly in the different experimental conditions. By studying serial individual trials when a new condition was begun, it was possible to evaluate how rapidly the postural muscle activity changes to deal with the new condition. There was sufficient variation from trial to trial that monotonic changes were not always found, but trends were evident.

Adaptation of EMG patterns to a new condition took place rapidly within only a few trials.

When the condition changed from unsupported to supported, EMG activity in leg and trunk muscles was already markedly reduced in the second movement and progressively diminished with successive trials (fig. 11).

\section{Discussion}

\section{ARM MUSCLES}

The duration of the first biceps burst in flexion movements is long $(127 \pm 9 \mathrm{~ms})$ compared to the durations of the other EMG components and to previously reported values. ${ }^{1923}$ Moreover, we have found that the first agonist burst duration increases with movement amplitude while former results have demonstrated that the duration of the first agonist 


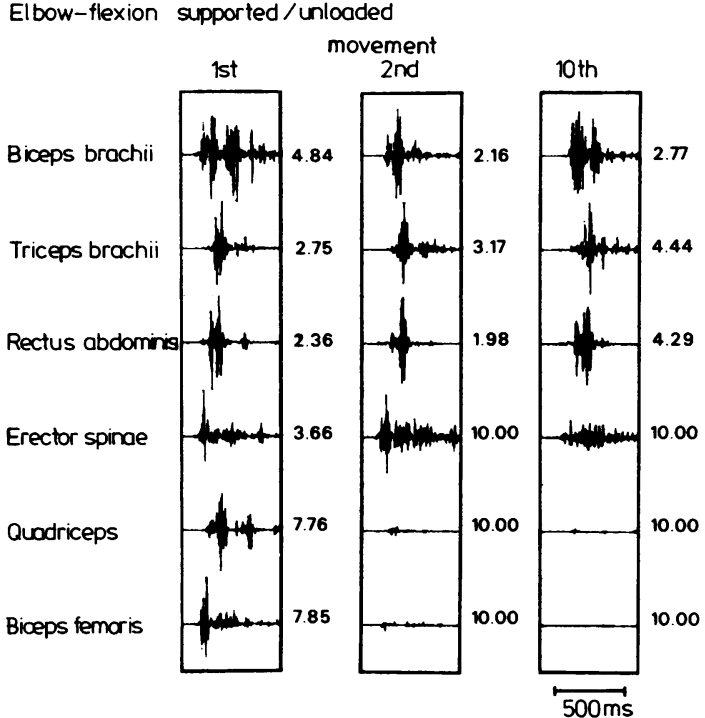

Fig 11 Adaptive behaviour of postural muscles with body support: first, second and tenth movement (from left to right) of a series of elbow flexions performed are shown. Postural activity in leg and trunk muscles diminishes with successive trials, and this is already apparent with the second movement. Note the increase of the scale factor for rectus abdominis in the tenth compared to former trials and larger magnitude of activity from biceps brachii in later trials. (Raw data from 1 subject).

and antagonist bursts are relatively constant for movements of various extent. In contrast to former experiments, the elbow movements analysed in our work are both of larger amplitude and performed in the sagittal plane against gravity. Since in elbow extensions of comparable amplitude the duration of the first agonist burst in triceps brachii was shorter (99 $\pm 10 \mathrm{~ms}$ ) and within the normal range, it is possible that the longer duration of the first biceps agonist burst is a consequence of having to overcome gravity in flexion movements. The increase of burst duration in parallel to the extent of elbow flexion is compatible with this concept since the larger the flexion movement in the sagittal plane, the more important becomes the vertical component and hence the gravitational force to be overcome.

Considerable triceps brachii activity overlaps with the first agonist burst in flexion movements. This overlapping may be due in part to the prolongation of the first agonist burst. Substantial overlapping of agonist and antagonist bursts was described during the acceleration phase of high speed movements. ${ }^{28}$ Such overlapping was shown not to be produced by current spread from agonists. ${ }^{29}$ Our subjects performed movements reaching peak velocities of $600^{\circ}$ per second, and hence this can be another reason for the overlap.

\section{POSTURAL MUSCLE ACTIVITY}

The EMG activity in various trunk and leg muscles associated with the fast voluntary elbow movements presumably has postural function with the role of maintaining stability of the body while standing. Our results indicate that some of this activity is anticipatory in nature, preceding the voluntary movement as described before by several investigators. ${ }^{13-17}$

One of the principal findings of the present study is the qualitative feature of alternating burst patterns in antagonist pairs of trunk and leg muscles, comparable to the triphasic pattern in the arm muscles. Although this type of activity has not been noted in the past, previous findings indicate the presence of alternating burst patterns in postural muscles (for example figs 3 and 4 of reference 17). Reasons which may explain the specific pattern observed in our experiments are: firstly, simplicity of the task by examining symmetrical arm movements, facilitating the recognition of postural antagonist pairs. Second, induction or enhancement of alternating burst patterns by free rapid movements, unrestricted by a tracking task or by a rigid experimental device. The burst pattern provides the classification of postural muscles into agonists and antagonists for that particular movement, the former being defined by their earlier onset time. The muscle which is the agonist indicates the direction of movement of the body segment (or the direction of force applied to the body segment); hence, for example, when erector spinae is the agonist this is associated with trunk extension. The pattern of activity includes an inhibitory period of the antagonist prior to agonist activation. This antagonist inhibition has been observed in arm muscles with rapid voluntary arm movements. ${ }^{2030}$ Inhibition of postural activity preceding activation of the prime mover has been described previously. ${ }^{14}$ In general, postural muscles precede the prime mover in a fixed sequence, leg muscles preceding trunk muscles, and the inhibitory period in the antagonists following the same distalto-proximal order. The distal-to-proximal order of postural activation has been reported in great detail. ${ }^{1516}$ Adjustments of the postural pattern are accomplished essentially in two ways: by variation of the timing of bursts relative to each other and by variation of burst magnitude, whilst there is no major change in burst duration. Lack of variation of burst duration is a general feature of rapid movements in normal man. ${ }^{19} 23$

We will refer to voluntary elbow movement as a "focal movement" and to the postural requirements as "postural set". The postural activity associated 
with a focal movement with a particular postural set is an "associated postural adjustment" (all three terms are in accordance with Cordo and Nashner ${ }^{15}$ ). Since the associated postural adjustment begins before the focal movement and is specific for the focal movement as well as the postural set, it must be pre-programmed. For example, the magnitudes of the first agonist and antagonist bursts in postural pairs and their timing with respect to the prime mover are adjusted to the event of load perturbation which comes up late during the focal movement. With respect to pattern changes which occur after the focal movement has started, it is not immediately clear whether they are related to central programs or whether they are produced by peripheral factors functioning in a feedback mode. For example, the distal-to-proximal order of activation is lost for later bursts of postural muscles with load perturbation in both the unsupported and supported state. Since these bursts occur after the onset of the load, it is possible that they are triggered by input from the arm disturbance. Postural responses evoked by limb perturbations have been identified. ${ }^{815163132}$ The latencies of these responses were in the range from 50 to $90 \mathrm{~ms}$ following the limb disturbance. It was concluded that these "interlimb reflexes" were centrally driven by afferent input from limb perturbation. In our experiment similar reflex responses might be expected subsequent to the onset of load perturbation. The second bursts of the postural agonists have latencies of 20 to $50 \mathrm{~ms}$ with respect to the onset of load, which seems to be a rather short period compared to the latencies of the interlimb reflexes mentioned above. Additionally, these second bursts are delayed from their ordinary time of appearance which is before the load disturbance, and this "inhibition" must be an anticipatory phenomenon. Hence, neither in the agonists nor in the antagonists was there consistent EMG activity which could be reliably identified with a reflex response. Nevertheless, this fact does not exclude peripheral mechanisms being involved in some modification of patterning of postural activity. This is compatible with interpretations of ballistic arm movements: that they are pre-programmed but modifiable.

\section{ADAPTATION}

Adaptation to a given postural $\operatorname{set}^{213}$ as well as to the properties of the focal movement is found to be a behaviour of associated postural adjustments. Adaptive changes concern both timing and magnitude of bursts of the postural pattern. Pairs of antagonist muscles may change their relationship to other pairs. Additionally, according to their role played in a specific task, bursts of postural antagon- ist pairs may change in magnitude in opposite directions, or alter their timing relative to each other.

Adaptation occurs remarkably rapidly. Changes in EMG patterns take place within only a few trials which is comparable to adaptation of functional stretch responses in leg muscles due to ankle perturbation if they are destabilising posture. ${ }^{16}$ Moreover, initial changes could already be apparent with the first trial of a new condition, without any experience, just with the knowledge of the condition. The presence of partially adjusted patterns already at the beginning of a new condition implies that the preprogrammed motor command is originally crudely prepared on the basis of expectation. ${ }^{33}$ Subsequently the motor commands are adjusted on the basis of sensory feedback information about the results obtained in previous trials, and this enables the system to optimize the performance.

INTERDEPENDENCE OF FOCAL MOVEMENT AND ASSOCIATED POSTURAL ADJUSTMENT

Associated postural adjustment and focal movement have to be linked to each other since the latter exerts forces to the body to be anticipated by postural activity in order to maintain stability. The temporal coupling of focal movement with associated postural adjustment is proven by our results. The relative timing of bursts is specific for a certain condition and is subject to variation with changes of the focal movement or alterations in the postural set, but does not depend upon the subject's reaction time. There is a proportional increase of the latency between the onset of postural and prime mover bursts with increasing postural requirements. This latency is least with body support which presumably contributes to the phenomenon of shortening of the focal movement's reaction time in this circumstance..$^{1516}$

Changes in magnitude of postural activity are found with different conditions. In general, the earlier the agonist is activated with respect to the prime mover, the larger will be its burst magnitude. The comparison of magnitudes of initial bursts of prime mover and postural muscles (fig 6 ) reveals clear differences between supported and loaded conditions. With body support biceps brachii usually shows larger magnitude than in the unsupported state while postural activity is markedly reduced. This reciprocal behaviour of postural activity and focal component was described by Cordo and Nashner ${ }^{15}$ and forms the basis of their theory about the organisation of postural adjustments. With the addition of load the magnitude of biceps brachii is in the same range regardless of body support (fig 6). This might be due to saturation of biceps activity with additional load. In the unsupported state with load perturbation increased activity is seen in both prime 
mover and postural agonists and decreased activity is seen in both triceps brachii and postural antagonists. Moreover, we find triceps brachii and postural antagonists to have a corresponding behaviour with changing the extent of elbow movement (fig 10). Hence, the agonist-antagonist principle presented here provides further insight into the process of specification of muscle synergy for postural stabilization associated with rapid arm movements. Postural mechanisms specify the balance of activity not only between postural and focal components but also between agonists and antagonists in both arm and postural muscles. While focal movement and associated postural adjustment are highly interdependent, the timing and magnitude of the associated postural adjustment will presumably be predictable on the basis of a general principle such as stability of the body.

Postural activity is an integral part of the motor program for voluntary movements. The question arises how voluntary movement and postural movement are linked together in the central nervous system. Postural activity with voluntary movement and that seen as a result of feedback are similar in the synergic pattern and in the time-course of adaptation. This suggests that voluntary movement and postural adjustments may be linked at a lower hierarchical level of the central nervous system. The appropriate postural pattern would be set-up during movement preparation in a feedforward mode in parallel with focal movement initiation. Basal ganglia and cerebellar systems seem to play roles in planning movements. Following the scheme proposed by Hallett and Khoshbin, ${ }^{34}$ basal ganglia may help to determine the appropriate muscles required to make the movement and their magnitudes while regulation of muscle timing may be a cerebellar function. In accord with this notion are the findings of Traub et $a l^{8}$ who found properly timed but reduced amplitude of "anticipatory postural responses" in patients with Parkinson's disease and the findings of Grimm and Nashner ${ }^{1}$ who have found inappropriately timed postural responses (called "functional stretch reflexes") in patients with cerebellar deficits.

This work was supported by grants from NIHR (23-P-55854/1), United Cerebral Palsy Research and The Charles A Dana Foundation Inc. Walter G Friedli was supported by a grant from the Schweizerische Stiftung für Medizinisch-Biologische Stipendien. Edward Bromfield participated in many of the early experiments. We are grateful to Lance Jackson, Roy Christopher, Johnson and John Ball for technical support, and Joseph Müller for photographic work.

\section{References}

' Grimm RJ, Nashner LM. Long Loop Dyscontrol. In: Desmedt JE, ed. Prog Clin Neurophysiol, vol. 4. Basel: Karger, 1978;70-84.

${ }^{2}$ Gurfinkel VS, Lipshits MI, Mori S, Popov KE. Postural reactions to the controlled sinusoidal displacement of the supporting platform. Agressologie 1976;17 B:71-76.

${ }^{3}$ Litvintsev AI. Mechanisms of man's vertical posture control. Agressologie 1973;14 B: 17-21.

${ }^{4}$ Litvintsev AI. System of man equilibrium control. Agressologie 1976;17 D:73-79.

${ }^{5}$ Nashner LM. Vestibular and reflex control of normal standing. In: Stein RB, Pearson KG, Smith RS \& Redford JB, eds. Control of Posture and Locomotion. New York: Plenum Press, 1973;291-308.

${ }^{6}$ Nashner LM. Adapting reflexes controlling the human posture. Exp Brain Res 1976;26:59-72.

7 Nashner LM. Fixed patterns of rapid postural responses among leg muscles during stance. Exp Brain Res 1977;30:13-24.

${ }^{8}$ Traub MM, Rothwell JC, Marsden CD. Anticipatory postural reflexes in Parkinson's disease and other akinetic-rigid syndromes and in cerebellar ataxia. Brain 1980;103:393-412.

${ }^{9}$ Coulmance M, Gahery Y, Massion J, Swett JE. The placing reaction in the standing cat: A model for the study of posture and movement. Exp Brain Res 1979;37:265-81.

${ }^{10}$ Gahery Y, Nieouilon A. Etude des réactions posturales accompagnant des mouvements provoqués par des stimulations corticales chez le chat (communication). $J$ Physiol (Paris) 1974;69:250 A.

" Massion J. Role of motor cortex in postural adjustments associated with movement. In: Asanuma $\mathrm{H} \&$ Wilson $\mathrm{V}$, eds. Integration in the Nervous System. Tokyo-New York: Igaku-Shoin, 1979;239-60.

${ }^{12}$ Regis H, Trouche E, Massion J. Movement and associated postural adjustment. In: Shahani M, ed. The Motor System: Neurophysiology and Muscle Mechanisms. Amsterdam: Elsevier, 1976:350-361.

${ }^{13}$ Belenkii VY, Gurfinkel VS, Paltsev YI. Elements of control of voluntary movements. Biofizika 1967; 12:154-61.

${ }^{14}$ Bouisset S, Zattara M. A sequence of postural movements precedes voluntary movement. Neurosci Lett 1981;22:263-70.

${ }^{15}$ Cordo PJ, Nashner LM. Properties of postural adjustments associated with rapid arm movements. $J$ Neurophysiol 1982;47:287-302.

${ }^{16}$ Nashner LM, Cordo PJ. Coordination of arm movements and associated postural adjustments in standing subjects. Soc Neurosci Abstr 1980;6:394.

17 Paltsev YL, Elner AM. Preparatory and compensatory period during voluntary movement in patients with involvement of the brain of different location. Biofizika 1967; 12:161-8.

${ }^{18} \mathrm{Ghez}$ C. Contributions of central programs to rapid movement in the cat. In: Asanuma $\mathbf{H} \&$ Wilson V, eds. Integration in the Nervous System. Tokyo-New York: Igaku-Shoin, 1979;305-20. 
${ }^{19}$ Hallett M, Marsden CD. Ballistic flexion movements of the human thumb.J Physiol (Lond) 1979;294:33-50.

${ }^{20}$ Hallett M, Shahani BT, Young RR. EMG analysis of stereotyped voluntary movements in man. $J$ Neurol Neurosurg Psychiatry 1975;38:1154-62.

${ }^{21}$ Angel RW. Electromyography during voluntary movement. The two-burst pattern. Electroencephalogr Clin Neurophysiol 1974;36:493-8.

22 Angel RW. Myoelectric patterns associated with ballistic movements: effect of unexpected changes in load. $J$ Human Movement Studies 1975;1:96-103.

${ }^{23}$ Freund $\mathrm{HJ}$, Büdingen $\mathrm{HJ}$. The relationship between speed and amplitude of the fastest voluntary contractions of human arm muscles. Exp Brain Res 1978;31:1-12.

${ }^{24}$ Garland H, Angel RW. Spinal and supraspinal factors in voluntary movement. Exp Neurol 1971;33:343-50.

${ }^{25}$ Wachholder K, Altenburger H. Beiträge zur Physiologie der willkürlichen Bewegungen. 10. Einzelbewegungen. Pfügers Arch ges Physiol 1926;214:642-61.

${ }^{26}$ Simon SR, Deutsch SD, Nuzzo RM, Mansour MJ, Jackson JL, Koskinen M, Rosenthal RK. Genu recurvatum in spastic cerebral palsy. $J$ Bone Joint Surg 1978; 60-A, 882-94.
${ }^{27}$ Simon SR, Nuzzo RM, Koskinen M. A comprehensive clinical system for four-dimensional motion analysis. Bull Hosp Joint Disease 1977;38:41-44.

${ }^{28}$ Lestienne F. Effects of inertial load and velocity of the braking process of voluntary limb movements. Exp Brain Res 1979;35:407-18.

${ }^{29}$ Bouisset S, Lestienne F, Maton B. The stability of synergy in agonists during the execution of a simple voluntary movement. Electroencephalogr Clin Neurophysiol 1977;42:543-51.

${ }^{30}$ Hufschmidt HJ, Hufschmidt T. Antagonist inhibition as the earliest sign of a sensory-motor reaction. Nature 1954;174:607.

${ }^{31}$ Kearney RE, Chan CWY. Interlimb reflexes evoked in human arm muscles by ankle displacement. Electroencephalogy Clin Neurophysiol 1981;52:65-71.

${ }^{32}$ Marsden CD, Merton PA, Morton HB. Anticipatory postural responses in the human subject. $J$ Physiol (Lond) 1978;275:47P-48P.

${ }^{33}$ Réquin J. Toward a psychobiology of preparation for action. In: Stelmach GE \& Réquin J, eds. Tutorials in Motor Behaviour. Amsterdam: Elsevier/NorthHolland Biomedical Press, 1980;1-26.

${ }^{34}$ Hallett M, Khoshbin S. A physiological mechanism of bradykinesia. Brain 1980;103:301-314. 